\title{
EFEITOS DO EXTRATO DE Eugenia uniflora SOBRE OS PARÂMETROS HEMATOLÓGICOS E PRESSÃO ARTERIAL DE RATOS HIPERTENSOS
}

\author{
Noemia Aparecida Partelli Mariani ${ }^{1}$ \\ Karen Silva Rangel ${ }^{2}$ \\ Emily Neves Souza ${ }^{3}$ \\ David Chaves Felicio Da Silva ${ }^{4}$ \\ Eduardo Frizzera Meira ${ }^{5}$ \\ Alessandra Simão Padilha ${ }^{6}$ \\ Juliana Aparecida Severi ${ }^{7}$ \\ Fabiana Dayse Magalhães Siman ${ }^{8}$
}

Resumo: A Eugenia uniflora é uma planta amplamente encontrada no Brasil, utilizada popularmente para tratar diversos distúrbios. O objetivo deste estudo foi avaliar os efeitos do extrato de suas folhas sobre parâmetros hematológicos e pressão arterial de ratos espontaneamente hipertensos (SHR). Os animais foram divididos em grupos controle e tratados com extrato nas doses de $100 \mathrm{mg} / \mathrm{kg}$ e $200 \mathrm{mg} / \mathrm{kg}$. 0 tratamento foi diário, via gavagem, por 28 dias. O sangue dos animais foi coletado semanalmente, para a contagem global e diferencial de leucócitos e ao final do tratamento, os animais foram anestesiados e foi realizada cateterização da artéria carótida para medida de pressão arterial sistólica, diastólica, média e frequência cardíaca. O extrato não alterou os parâmetros hematológicos e não reduziu a frequência cardíaca dos SHR, porém observou-se uma tendência à redução. Ao passo que, diminuiu a pressão arterial sistólica, diastólica e média, sugerindo um efeito anti-hipertensivo.

Palavras-chave: Eugenia uniflora; Parâmetros hematológicos; Pressão arterial, SHR.

\footnotetext{
${ }^{1}$ Farmácia/Universidade Federal do Espírito Santo - Centro de Ciências Exatas Naturais e da Saúde, Brasil. Email: noemiapartelli@hotmail.com.

${ }^{2}$ Farmácia/Universidade Federal do Espírito Santo - Centro de Ciências Exatas Naturais e da Saúde, Brasil. Email: karenrangelfarm@gmail.com.

3 Farmácia/Universidade Federal do Espírito Santo - Programa de Pós-Graduação em Ciências Fisiológicas, Universidade Federal do Espírito Santo, Brasil. E-mail: neves.emilly@hotmail.com.

${ }^{4}$ Farmácia/Universidade Federal do Espírito Santo - Programa de Pós-Graduação em Ciências Fisiológicas, Universidade Federal do Espírito Santo, Brasil. E-mail: felicio762@gmail.com.

5 Farmácia/Universidade Federal do Espírito Santo - Programa de Pós-Graduação em Ciências Fisiológicas, Universidade Federal do Espírito Santo, Brasil. E-mail: eduardo.frizzera.meira@gmail.com.

6 Farmácia/Universidade Federal do Espírito Santo - Programa de Pós-Graduação em Ciências Fisiológicas, Universidade Federal do Espírito Santo, Brasil. E-mail: ale_padilha@hotmail.com.

${ }^{7}$ Farmácia/Universidade Federal do Espírito Santo - Centro de Ciências Exatas Naturais e da Saúde, Brasil. Email: juseveri@yahoo.com.br.

${ }^{8}$ Farmácia/Universidade Federal do Espírito Santo - Centro de Ciências Exatas Naturais e da Saúde, Brasil. Email: famisiman@gmail.com.
} 